\title{
Binding free energies of small-molecules in phospholipid membranes: aminoacids, serotonin and melatonin
}

\author{
Jordi Marti`, Huixia Lu \\ ${ }^{a}$ Department of Physics, Technical University of Catalonia-Barcelona Tech, B4-B5 \\ Northern Campus UPC. Jordi Girona, 1-3. 08034 Barcelona. Catalonia. Spain.
}

\begin{abstract}
Binding free energy barriers of small-molecules adsorbed at phospholipid membranes have been computed for three aminoacids, serotonin and melatonin for a di-palmitoyl-phosphatidil-choline membrane in aqueous sodium chloride solution. From potentials of mean force we observed that molecular probes were fully solvated by water or embedded into the interface of the membrane. When bound to water, barriers were between 0.2 and $1.75 \mathrm{kcal} / \mathrm{mol}$ but when associated to lipids, range was between 0.6 and $3.1 \mathrm{kcal} / \mathrm{mol}$. All species but histidine were usually bound to oxygens from phosphate and glycerol groups. No free permeation through the membrane of any small-molecule was observed.

Key words: Helmholtz free energy, phospholipid membrane, small-molecule, Molecular Dynamics.
\end{abstract}

\section{Introduction}

The principal components of human cellular membranes are phospholipids, cholesterol and proteins, all of them embedded in a salty water solution. Phospholipid membranes provide the framework to biomembranes and they consist 5 of two leaflets of amphiphilic lipids which are molecules with a hydrophilic head and one or two hydrophobic tails [1]. The fluidity of the membrane is mainly regulated by the amount of cholesterol, in such a way that membranes with

\footnotetext{
${ }^{*}$ Corresponding author

Email addresses: jordi.marti@upc.edu, huixia.lu@upc.edu (Jordi Marti, Huixia Lu)
} 
high cholesterol contents are stiffer than those with low amounts but keeping the appropriate fluidity for allowing normal membrane functions.

In this letter we have focussed our efforts in the study of zwitterionic phospholipid membranes that can help understand basic biological membrane functions and its interaction with the environment. As an example of a prototype membrane, the one formed by di-palmitoyl-phosphatidyl-choline (DPPC) is one of most relevant of all, being a major constituent (about 40\%) of pulmonary lungs 2. Further, human lungs are coated with a lattice-like structure formed by protein and lipid mixture called lung surfactant, preventing the lungs from collapsing and protecting us from bacterial and viral infections. A large number of simulations have already been performed on DPPC, often including the influence of cholesterol in water environments 3 . On the other hand, the role 20 of proteins and drugs and their interactions with the membrane structure is undoubtedly a relevant field of research. In this work we have considered the introduction into the lipid bilayer structure of small biological probes of different kinds: three aminoacids (tryptophan[4] (TRP), histidine-E (HIS) and tyrosine (TYR), the neurotransmitter serotonin[5] (SRO) and the hormone melatonin [6] 25 (MEL).

Aminoacids are organic compounds containing amine (-NH2) and carboxyl (-COOH) functional groups, along with a side chain (R group) specific to each amino acid. It is well known that aminoacids can either be essential, i.e. indispensable or non-essential. An essential aminoacid cannot be synthesized de novo by the organism and it would be necessarily supplied by the diet. The nine aminoacids humans cannot synthesize are: phenylalanine, valine, threonine, tryptophan, methionine, leucine, isoleucine, lysine, and histidine.

Tryptophan is able to act as a building block in protein biosynthesis, while proteins are fundamentals required to sustain life. In addition, it helps in the 35 regulation of human sleep. In turn, histidine is an alpha-aminoacid that is also used in the biosynthesis of proteins. It is positively charged at physiological pH. Initially thought essential only for infants, longer-term studies have shown it is essential for adults also. Differently, tyrosine is another of the 20 standard 
aminoacids that are used by cells to synthesize proteins, but it is non-essential.

40 Tyrosine is required for the synthesis of the neurotransmitter dopamine. We selected these three particular amino acids to explore whether or not their interactions with zwitterionic cell membranes are related to their essential or non-essential characteristics.

Serotonin is a neurotransmitter biochemically derived from tryptophan and

45 it is primarily found in the gastrointestinal tract, blood platelets and at the central nervous system of animals, including humans. It is thought to be a contributor to the regulation of human mood and happiness. Serotonin can be converted to melatonin (a neurohormone), that may help humans to the regulation of biological rhythms, to induce sleep, to work as a strong antioxidant 50 and also contribute to the protection of the organism from carcinogenesis and neurodegenerative disorders such as Alzheimer's disease [7.

In summary, given the importance of aminoacids, neurotransmitters and hormones for the correct function of the body, we have explored their interactions with a prototypical cell membrane formed by DPPC and water in a ${ }_{55}$ salty solution of sodium chloride using all-atom molecular dynamics (MD) simulations, analyzing its local structure through free energy profiles based on the reversible work theorem. Some previous studies indicated strong interactions of serotonin with di-myristoil-phospahtidyl-choline (DMPC) and di-oleoylphosphatidyl-choline (DOPC) membranes 8 or of some neurotransmitters with 60 DPPC [9].

We provide the details of the simulations in section 2 and explain the main results of the work in section 3 , focusing our attention especially on the free energy barriers of the adsorption of small-molecule species. Finally, some concluding remarks are outlined in section 4. 


\section{Methods}

A model of a zwitterionic lipid bilayer membrane in aqueous sodium chloride solution has been build by means of the CHARMM-GUI tool 10, 11. The membrane was composed by: 204 lipids, distributed in two leaflets of 102 flexible DPPC $\left(\mathrm{C}_{40} \mathrm{H}_{80} \mathrm{NO}_{8} \mathrm{P}\right)$ molecules, surrounded by TIP3P 12] water (W) molecules (enough to ensure full hydration in all cases), with 17 sodium and 17 chlorine ions, corresponding to physiological concentration, plus one small-molecule. In order to compare several probes of different chemical structures being able to perform a variety of biological functions, we considered five species. They are three aminoacids: tryptophan $\left(\mathrm{C}_{11} \mathrm{H}_{12} \mathrm{~N}_{2} \mathrm{O}_{2}\right)$; histidine-E $\left(\mathrm{C}_{6} \mathrm{H}_{9} \mathrm{~N}_{3} \mathrm{O}_{2}\right)$ and tyrosine $\left.\mathrm{C}_{9} \mathrm{H}_{11} \mathrm{NO}_{3}\right)$, one neurotransmitter, serotonin $\mathrm{C}_{10} \mathrm{H}_{12} \mathrm{~N}_{2} \mathrm{O}$ ) and a hormone, melatonin $\left(\mathrm{C}_{13} \mathrm{H}_{16} \mathrm{~N}_{2} \mathrm{O}_{2}\right)$.

Sketches of the backbone structure of the small molecules and DPPC are represented in Fig.1. Each molecule was described with atomic resolution. MD simulations were performed with the NAMD2 simulation package[13] at a fixed temperature of $323.15 \mathrm{~K}$ and at the averaged pressure of $1 \mathrm{~atm}$. At this temperature, the DPPC membrane is fully at the liquid-disordered state (see for instance Refs. [14, 15]). The temperature was controlled by a Langevin thermostat [16] with a damping coefficient of $1 \mathrm{ps}^{-1}$.

We considered all systems at the the isobaric-isothermal ensemble. i.e. at constant number of particles $(\mathrm{N})$, pressure $(\mathrm{P})$ and temperature $(\mathrm{T})$ conditions, with equilibration periods for all simulations of more than $40 \mathrm{~ns}$. After equilibration, we recorded statistically meaningful trajectories of more than $60 \mathrm{~ns}$. A typical size of the system was of $80 \AA \times 80 \AA \times 81 \AA$, regardless of the probe considered, since the biggest part of the membrane was made of the same components, i.e. DPPC, water and ions in exactly the same concentrations. The simulation time step was set to $2 \mathrm{fs}$ in all cases. We considered the CHARMM36 force field [17, 18, which is able to reproduce the area per lipid in excellent agreement with experimental data. All bonds involving hydrogens were fixed to constant length, allowing fluctuations of bond distances and all sorts of angles 

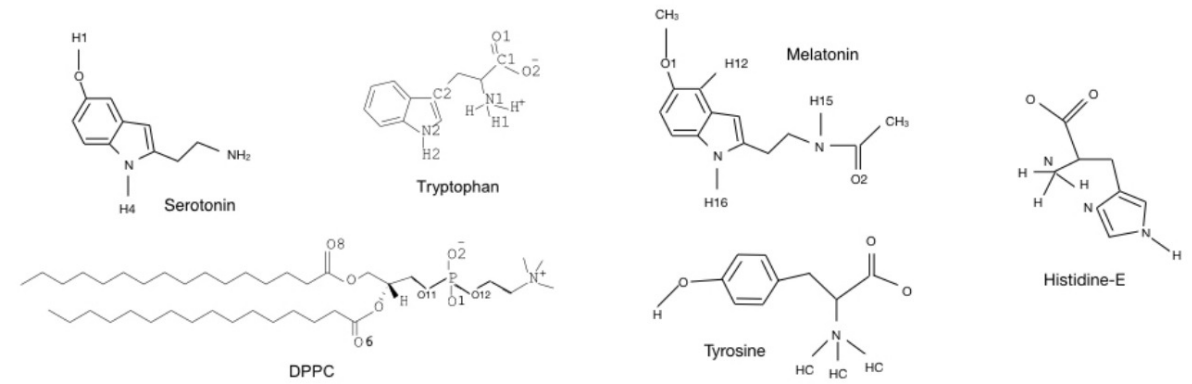

Figure 1: Sketches of the backbone structures of DPPC, L-tryptophan, histidine-E, tyrosine, serotonin and melatonin. Hydrogens bound to carbon not shown. The highlighted sites of the small-molecules (TRP, TYR, HIS, MEL, SRO) and of DPPC will be referred in the text by the labels defined here. Due to the zwitterionic characteristics of L-tryptophan, its site H1 corresponds to any of the three hydrogens bound to N1, which share the positive charge. Same case for the three ' $\mathrm{HC}$ ' sites of tyrosine and the triplet ' $\mathrm{H}$ ' bound to nitrogen in histidine. Sites $\mathrm{O} 1$ and $\mathrm{O} 2$ of TRP share the negative charge, exactly the same as in tyrosine and histidine.

for the remaining atoms. Van der Waals interactions were cut off at $12 \AA$ with a smooth switching function starting at $10 \AA$ A. Long ranged electrostatic forces were taken into account by means of the particle mesh Ewald method[19], with a grid space of about 1 Å. Electrostatic interactions were updated every time step. Finally, periodic boundary conditions were applied in all directions.

As a general fact, we did not observe any natural permeation of a small molecule across the DPPC membrane (from one interface to the pther) at the time scale of our simulations, in agreement with the findings of Wood et al. for serotonin and tryptophan adsorbed at a 1-palmitoyl-2-oleoyl-phosphatidylcholine (POPC) membrane 20]. This would be in qualitative agreement with the reported work of Kell et al.21] on pharmaceutical drug permeation, who stated that diffusion of a small-molecule or drug through a cell membrane can only happen by means of the help of some mediating-carrier. Nevertheless, other authors such as Di et al. 22 reported evidence of pure diffusion of small drugs across membranes, like in the case of brain-blood barrier permeation of lipophilic small-molecules [23]. From our findings we cannot support any of these 
results, essentially due to the limited length of our calculations in the range of $100 \mathrm{~ns}$, whereas some diffussion processes may occur at longer time scales.

\section{Results and discussion} under wrong assumptions due to artificial undulations of the membrane sets. The best estimation for the liquid-phase was of $64 \AA^{2}$. In a quite recent work, Kučerka et al. [15] found a value of $63.1 \AA^{2}$ for DPPC at $323.15 \mathrm{~K}$ by means of X-ray and neutron scattering techniques.

135

Once the local structure around a small-molecule (for instance, a set of radial distribution functions $g(r)$ ) has been determined, a common way to analyze the microscopic forces relevant for the binding process is obtaining the Helmholtz free energy by means of the so-called potential of mean force (PMF) between system. We have monitored the surface area per lipid considering the total surface along the $X Y$ plane (plane parallel to the bilayer surface) divided by the number of lipids in one lamellar layer 24]. The final averaged areas per lipid are reported in Table1. The reader should note that this values for the area per lipid arise naturally from the relaxation of the system at a given temperature, pressure and number of particles rather than being an imposition to fit the experimental value. We needed to consider time scales of more than $40 \mathrm{~ns}$ in order to obtain converged area per lipid in all cases. The main trend observed is that all values are very close to $61 \AA^{2}$, in overall good agreement with other computational in a wide variety of thermodynamical conditions [25, 26, 27, 28, 29] where the values for pure DPPC range between 50 and $63 \AA^{2}$. From the experimental side, an influential review from Nagle et al. 30 reported values of the area per lipid of pure DPPC membranes obtained from a wide variety of methods (NMR, X-ray and neutron scattering) between 56 and $72 \AA^{2}$ at the liquid phase ( $T>323 \mathrm{~K})$. It should be pointed out that some of these values were measured particles 1 and 2, namely $W_{12}(r)$, that can be readily obtained from the $g_{12}(r)$

As a very first primary output we have computed the area per lipid for each 
Table 1: Area per lipid of the membrane systems. Estimated errors are in parenthesis.

\begin{tabular}{c|c}
\hline Small-molecule & $A\left(\AA^{2}\right)$ \\
\hline L-Tryptophan & $61.5(1.0)$ \\
Histidine-E & $60.5(2.0)$ \\
Tyrosine & $60.8(1.9)$ \\
Serotonin & $61.2(1.8)$ \\
Melatonin & $61.0(1.0)$ \\
\hline
\end{tabular}

given by:

$$
g_{12}(r)=\frac{V\left\langle n_{2}(r)\right\rangle}{4 N_{2} \pi r^{2} \Delta r},
$$

where $n_{2}(r)$ is the number of atoms of species 2 surrounding a given atom of species 1 inside a spherical shell of width $\Delta r . V$ stands for the total volume and $N_{2}$ is the total number of particles of species $2 . W_{12}(r)$ is the reversible work required to move two tagged particles from infinite separation to a relative separation $r$ (see for instance Ref.[31, chapter 7):

$$
W_{12}(r)=-\frac{1}{\beta} \ln g_{12}(r),
$$

where $\beta=1 /\left(k_{B} T\right)$ is the Boltzmann factor, $k_{B}$ the Boltzmann constant and $\mathrm{T}$ the temperature. This remarkable theorem can be proved by considering the averaged force between two particles ' 1 ' and '2' inside the remaining system (particles ' 3 ', ' 4 ',...'N') acting as the solvent. The average (over all configurations) force between particles '1' and '2' fixed at their corresponding positions is given by:

$$
\begin{aligned}
-\left\langle\frac{d U(r)}{d \vec{r}_{1}}\right\rangle_{\vec{r}_{1}, \vec{r}_{2}} & =-\frac{-\int d \vec{r}_{3} \cdots d \vec{r}_{N}\left(\frac{d U(r)}{d \vec{r}_{1}}\right) e^{-\beta U}}{\int d \vec{r}_{3} \cdots d \vec{r}_{N} e^{-\beta U}}= \\
& =\beta \frac{\frac{d U(r)}{d \vec{r}_{1}} \int d \vec{r}_{3} \cdots d \vec{r}_{N} e^{-\beta U}}{\int d \vec{r}_{3} \cdots d \vec{r}_{N} e^{-\beta U}}=
\end{aligned}
$$




$$
\begin{aligned}
& =\beta \frac{d}{d \vec{r}_{1}} \ln \int d \vec{r}_{3} \cdots d \vec{r}_{N} e^{-\beta U} \\
& =\beta \frac{d}{d \vec{r}_{1}} \ln \left[\left(N(N-1)\left(\frac{\int d \vec{r}_{3} \cdots d \vec{r}_{N} e^{-\beta U}}{\int d \vec{r}_{1} \cdots d \vec{r}_{N} e^{-\beta U}}\right)\right]=\right. \\
& =\beta \frac{d}{d \vec{r}_{1}} \ln g\left(\vec{r}_{1}, \vec{r}_{2}\right),
\end{aligned}
$$

where the definition of the radial distribution function from the statistical point of view has been assumed (see Ref. 31]). Eq3 3 reveals that, since the force at its left side is the change in Helmholtz free energy as a function of $\vec{r}_{1}$, such free energy is given by Eq2

The use of a variety of methods to compute the PMF has been extensively discused in the literature, as it was reported for instance in Ref. 32], where up to twelve methods based on one-dimensional coordinates were applied to the benckmark case of a methane pair in aqueous solution. The authors concluded that the best choice is a constraint-bias simulation combined with force averaging for Cartesian or internal degrees of freedom. The results from unbiased simulations, as those reported in the present work, were considered good at the qualitative level, with the PMF reasonably well reproduced. However, the use of one-dimensional reaction coordinates is simply an approximation to the real ones 33, which may be in general multidimensional, presumably involving a limited number of water molecules and, eventually coordinates or distances to the other species of the system. Methods which do not assume any preconceived reaction coordinates such as transition path sampling [34, 35, 36] or, those allowing to consider several complementary collective variables, such as metadynamics 37] would be in order to obtain much more accurate free energy landscapes for TRP adsorption, but they require a huge amount of computational time. So, since the determination of the true reaction coordinate for the adsorption of small-molecules at zwitterionic membranes is out of the scope of this paper, we will consider the radial distances between two species as our order parameters useful to work as reaction coordinates of unbiased simulations. Another standard tool such as umbrella sampling, which allows to place the probe at different $z$ coordinates (where $Z$ is the axis perpendicular to the sur- 
face of the membrane) and hints the free energy difference for probe adsorption as a function of his location inside the membrane has not been considered here since we observed that small molecules never cross the membrane by means of pure diffusion (passive transport) at the time scale of our simulations (hundred nanoseconds), in agreement with some findings on drug transport through membranes 21]. In summary, our hypothesis is that the relative distance between the small-molecule and a water (or lipid) site is a useful order parameter able to describe the relative Helmholtz free energies for ion pairing, but it is not necessarily the best reaction coordinate to descvribe such molecular binding.
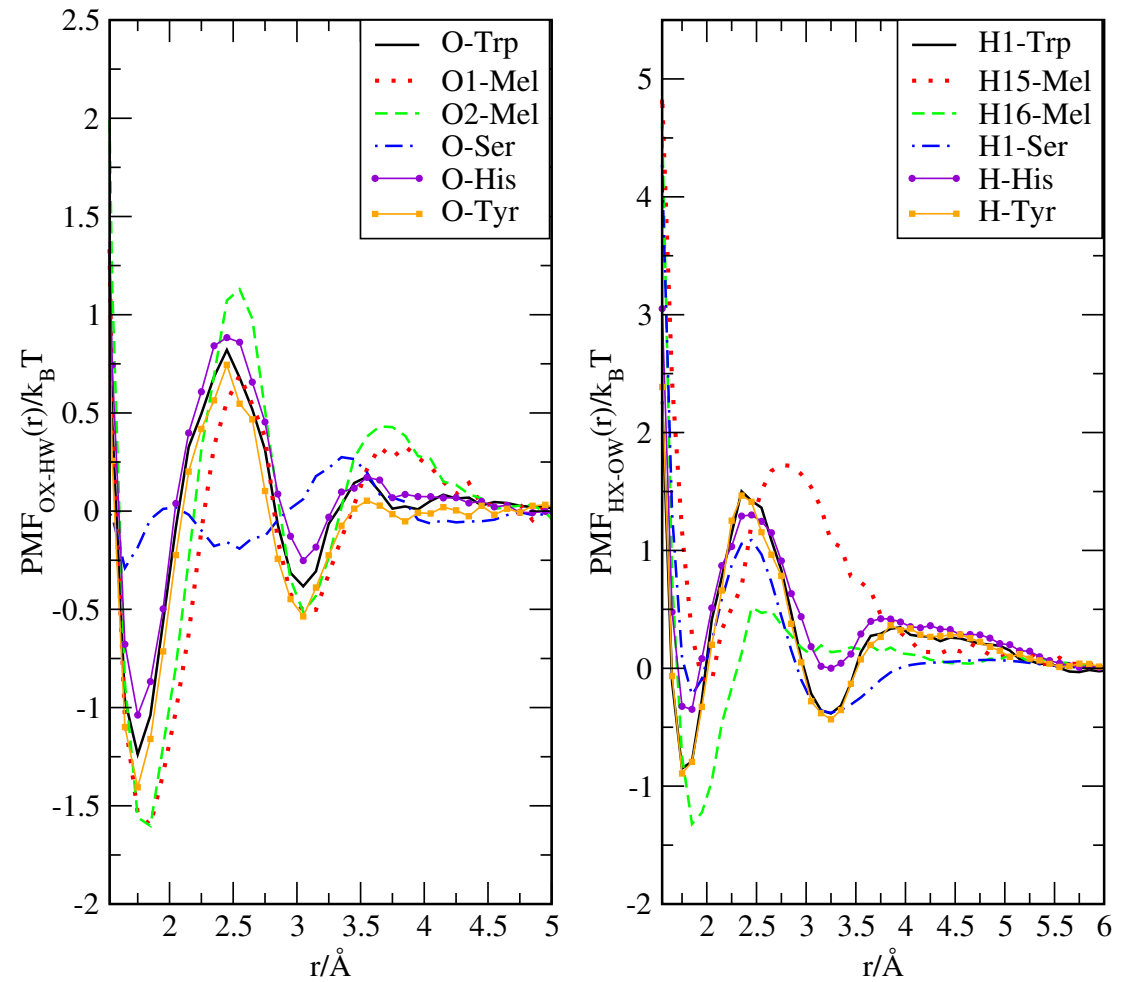

Figure 2: Potentials of mean force for the adsorption of small-molecules to water molecules. Oxygens of aminoacids (TRP, HIS, TYR) share the negative charge as indicated in Fig 1 . whereas hydrogens of these aminoacids are those forming triplets bound to nitrogen atoms.

The results of water and DPPC versus small-molecule PMF are displayed in 
Figures 2 (water) and 3 (DPPC) in units of $k_{B} T$. In order to quantify the height of all barriers, we included the corresponding numerical estimation in Table 2 assuming that, for the present simulations, $1 k_{B} T=0.64185 \mathrm{kcal} / \mathrm{mol}$. The values reported in Table 2 are in between 0.2 and $3.1 \mathrm{kcal} / \mathrm{mol}$, i.e. of the same order of magnitude of the free energies of adsorption of metal ions in DMPC membranes 38.

195

We show PMF for water's oxygens at the plots in left column and those for water's hydrogens in plots at the right side of Fig 2 For oxygens of water a free energy barrier is seen in all cases, defined by a neat first minimum and a second minimum clearly defined, although the barrier of water-serotonin is much smaller than those corresponding to the rest of pairings. This finding is in good agreement with the results reported by Wood et al. 20, indicating that serotonin is normally anchored to the POPC membrane whereas TRP and other zwitterions have full access to the water region. In the case of hydrogens of water, the second minimum is not well defined for melatonin's hydrogens 'H15' and 'H16' (see Fig.1). The binding of small-molecules to water reveals, as a general fact, free energy barriers of between $0.3-2.7 k_{B} T$ with stable binding distances very close to the typical hydrogen-bond (HB) distances in water, given by the position of the first minimum of the oxygen-hydrogen radial distribution function $(1.85 \AA$ ) [39]. However, the typical energy of water-water HBs estimated from ab-initio calculations is of about $5 \mathrm{kcal} / \mathrm{mol}[40$, value significantly larger than those observed in this work.

A closer look indicates that the largest barriers correspond to HB formed by oxygens of a small-molecule (acting as acceptors) and hydrogens of water, acting as donors. "Reverse" hydrogen-bonding composed by hydrogens of a small-molecule (donors) and oxygens of water (acceptors) is also possible but 215 it is weaker than the former, with significantly smaller free energy barriers, up to $2.3 k_{B} T$ in size. In summary, the aqueous solvation of small probes revealed similar characteristics regardless of the type of the molecule (aminoacid, neurotransmitter, hormone), with strongest pairing for melatonin-water and weakest for serotonin-water. 
Table 2: Free energy barriers $\Delta \mathrm{F}$ (in $\mathrm{kcal} / \mathrm{mol}$ ) for the binding of small-molecules to water and to DPPC.

\begin{tabular}{c|c|c|c|c}
\hline Probe (Active site) & O-water & H-water & O2-DPPC & O8-DPPC \\
\hline Tryptophan (O) & - & 1.32 & - & - \\
Tryptophan (H1) & 1.50 & - & 2.68 & 1.81 \\
Tryptophan (H2) & - & - & 1.93 & 1.00 \\
Histidine (O) & - & 1.24 & - & - \\
Histidine (H) & 1.06 & - & - & - \\
Tyrosine (O) & - & 1.37 & - & - \\
Tyrosine (H) & 1.51 & - & 3.13 & 3.01 \\
Tyrosine (HC) & - & - & 2.79 & 1.51 \\
\hline Serotonin (O) & - & 0.20 & - & - \\
Serotonin (H1) & 0.82 & - & 1.90 & 1.48 \\
Serotonin (H4) & - & - & 1.71 & 1.66 \\
Melatonin (O1) & - & 1.44 & - & - \\
Melatonin (O2) & - & 1.75 & - & - \\
Melatonin (H15) & 1.17 & - & - & 1.22 \\
Melatonin (H16) & 1.16 & - & 1.91 & 0.58 \\
\hline
\end{tabular}


Regarding the interactions of small-molecules versus lipid atomic's sites and from data reported in Table 2 and in Fig 3 , we observe the highest barrier (3.13 $\mathrm{kcal} / \mathrm{mol}$ ) corresponding to the pairing of TYR (through its hydroxil's hydrogen) with the phosphate oxygen 'O2' of DPPC. In decreasing order, 'HC' of TYR and 'H1' of TRP show also strong interactions with 'O2'. From a general point of view, all small molecules but histidine are able to establish $\mathrm{HB}$ with 'O2' and also with the site 'O8' of DPPC, much deeper in the membrane (see Fig.1). It should be pointed out that the barrier of TYR to ' $\mathrm{O} 8$ ' is remarkable, of about 3 $\mathrm{kcal} / \mathrm{mol}$ and further indicates the stability of TYR at the membrane, compared to likes of the remaining aminoacids (HIS, TYR), melatonin and serotonin.

The most stable distance for 'O2' in DPPC bound to TRP is of about 1.75 $\AA$, i.e. the position of the first minimum of the PMF between TRP and DPPC. As it has been stateted before, such distance is of the order of the typical HB distance in water. For the sake of comparison, the PMF of TRP in a dioleoylphosphatidylcholine bilayer membrane shows a barrier of the order of 4 $\mathrm{kcal} / \mathrm{mol}$ [1], whereas the barrier for the movement of TRP (attached to a polyleucine $\alpha$-helix) inside a DPPC membrane was reported to be of $3 \mathrm{kcal} / \mathrm{mol}[6]$. Finally, neurotransmitters such as glycine, acetylcholine or glutamate were reported to show small barriers of about $2-5 \mathrm{~kJ} / \mathrm{mol}$ when located close to the lipid glycerol backbone 9 .

240 In order to have a more detailed idea on the particular binding of some small-molecules to the membrane, we are reporting two characteristic snapshots of SRO and TYR linked to two DPPC molecules (see Fig,4). There we can observe that the most active sites are hydrogens belonging to hydroxil groups, bound to DPPC at different sites ('O2' and 'O8' simultaneously for TYR, right and 'O2' for SRO, left). These images are only significant configurations selected among a wide variety of possible choices (see Table 2) and may help the reader to enlighten the relatively complex multiple hydrogen-bonding connections between the small-molecules and DPPC described above. 


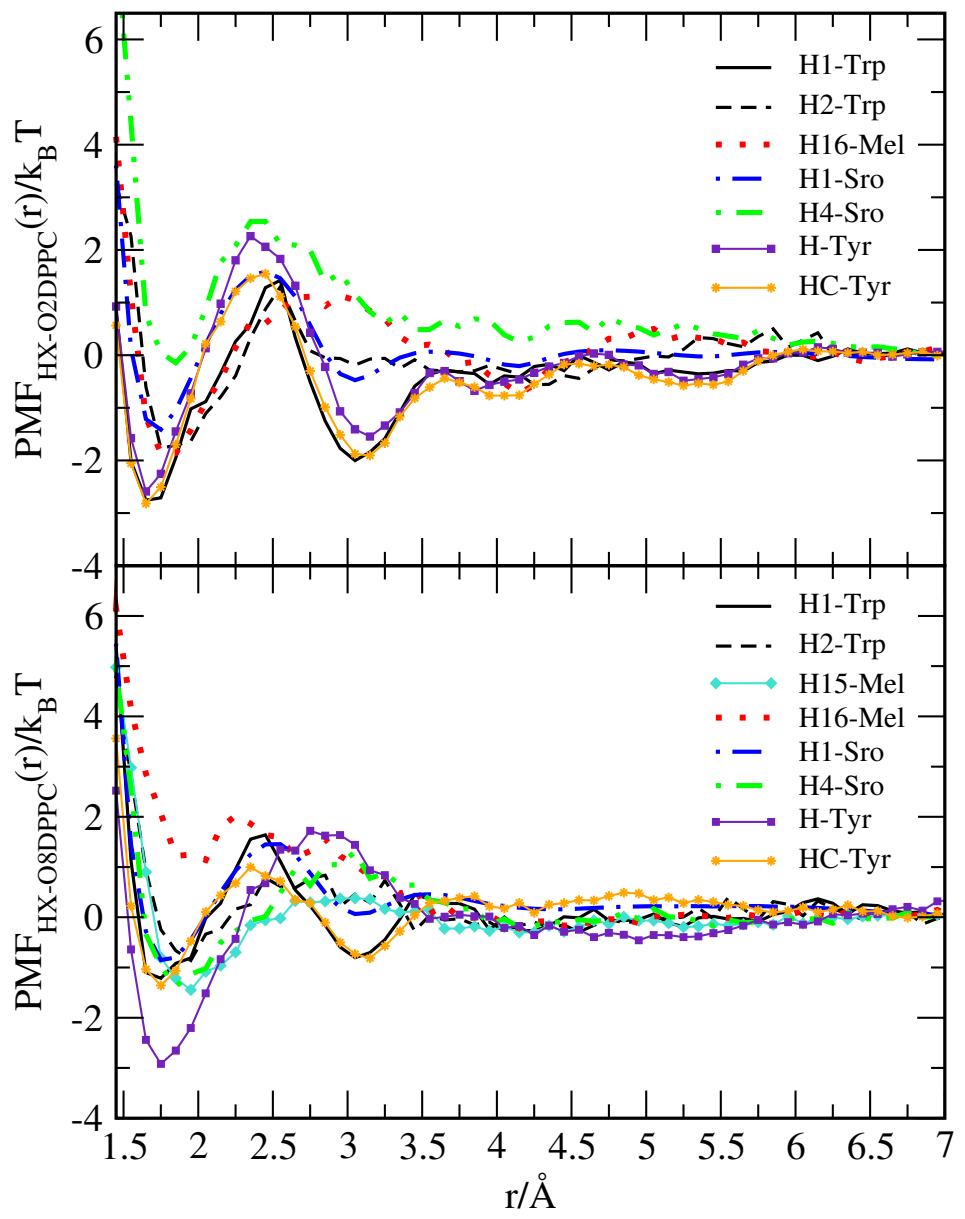

Figure 3: Potentials of mean force for DPPC-small molecules.

\section{Conclusions}

A series of molecular dynamics simulations of a DPPC lipid bilayer membrane in aqueous ionic solution of $\mathrm{NaCl}$ with an embedded single small-molecule have been performed by MD using the CHARMM36 force field at the canonical ensemble at $323 \mathrm{~K}$. We have first focused our analysis on the characterization of the different setups and found that the area per lipid are practically not influenced by the presence of the particular probe and they are in all cases around $61 \AA^{2}$, in agreement with other computational and experimental data. 

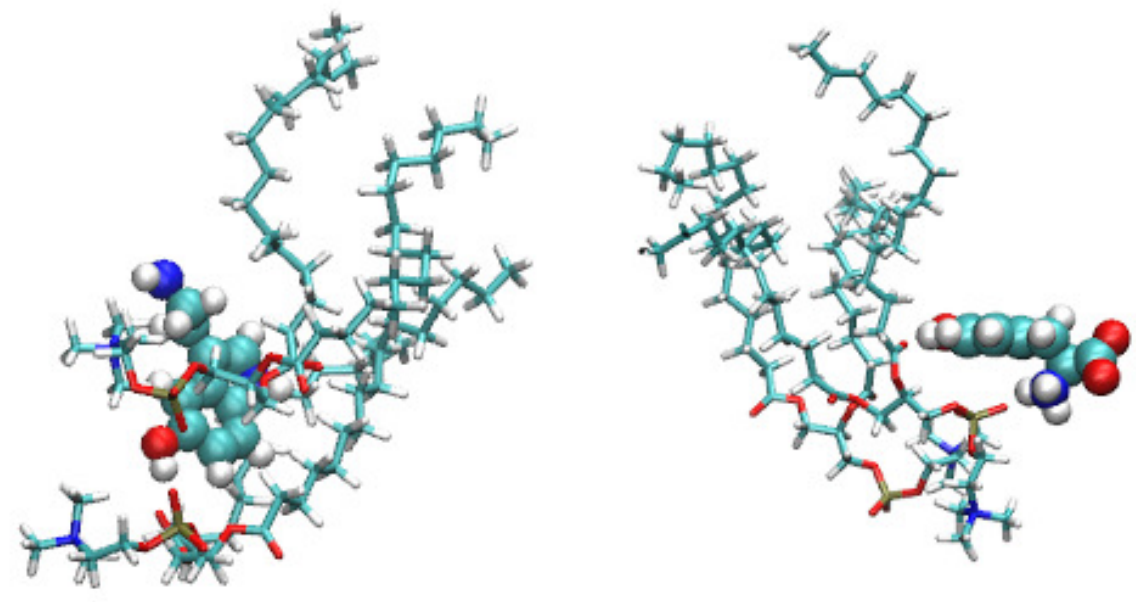

Figure 4: Snapshots of typical small-molecule and DPPC bonds. SRO-DPPC (left) and TYRDPPC (right).

The Helmholtz free energy of adsorption has been evaluated through the potentials of mean force. We have considered the usual one-dimensional reaction coordinates based on atomic distances for selected sites. We chose six types of particles: (1) the hydrogens labeled 'H1' and (2) the double bonded oxygens 'O1' and 'O2' of the small molecule; (3) the three water sites; (4) the charged oxygens labeled 'O2' and 'O8' of DPPC. Our data revealed the existence of a strong first coordination shell and a milder second coordination shell for small moleculewater structure, which correspond to two minima in the corresponding PMFs, with energy barriers for TRP-water association of the order of 1-2 kcal/mol. Conversely, the binding to DPPC involves a single coordination shell for the two sites of possible association (oxygens 'O2' and 'O8' of DPPC versus hydrogens in the small-molecules) and energy barriers between 1-3 kcal/mol.

Throughout our simulation runs, we did not observe any event of permeation 270 of a small-molecule across the DPPC membrane. This is in agreement with previous results for serotonin and tryptophan adsorbed at a 1-palmitoyl-2-oleoylphosphatidyl-choline (POPC) membrane[20, suggesting that neither SRO nor 
TRP would be able to cross the blood-brain barrier without the participation of some especific mediating carrier. Concering the essentiallity of the two amino acids reported in the present work (HIS, TRP), we observed that TRP is able to enter the interfacial membrane, whereas HIS is not. Interestingly, tyrosine, a non-essential aminoacid shows the highest free energy barriers, indicating that it is the most stable molecule for DPPC binding. Serotonin has revealed to be a molecule anchored at the membrane and with a low propensity to be solvated by water, whereas its derivative melatonin is able to equally interact with water and DPPC, showing similarly strong free energy barriers.

\section{Acknowledgments}

The authors gratefully acknowledge financial support provided by the Spanish Ministry of Economy and Knowledge (grant FIS2015-66879-C2-1-P). HL is the recipient of a grant from the Chinese Scholarship Council (grant 201607040059). Computational resources awarded by the Barcelona Supercomputing CenterSpanish Supercomputing Network (grant FI-2018-2-0018) are also acknowledged.

\section{References}

[1] O. G. Mouritsen, Life-As a Matter of Fat, Springer, 2005.

[2] S. Schurch, M. Lee, P. Gehr, Pulmonary surfactant: Surface properties and function of alveolar and airway surfactant, Pure Appl. Chem. 64 (11) (1992) 1745-1750.

[3] G. S. Ayton, G. A. Voth, Mesoscopic lateral diffusion in lipid bilayers, Biophys. J. 87 (5) (2004) 3299-3311.

[4] K. M. Sanchez, G. Kang, B. Wu, J. E. Kim, Tryptophan-lipid interactions in membrane protein folding probed by ultraviolet resonance raman and fluorescence spectroscopy, Biophys. J. 100 (9) (2011) 2121-2130. 
[5] A. Slominski, I. Semak, A. Pisarchik, T. Sweatman, A. Szczesniewski, J. Wortsman, Conversion of l-tryptophan to serotonin and melatonin in human melanoma cells, FEBS letters 511 (1-3) (2002) 102-106.

[6] A. J. de Jesus, T. W. Allen, The role of tryptophan side chains in membrane protein anchoring and hydrophobic mismatch, BBA-Biomembranes 1828 (2) (2013) 864-876.

[7] I. Kostoglou-Athanassiou, Therapeutic applications of melatonin, Therapeutic advances in endocrinology and metabolism 4 (1) (2013) 13-24.

[8] G. H. Peters, C. Wang, N. Cruys-Bagger, G. F. Velardez, J. J. Madsen, P. Westh, Binding of serotonin to lipid membranes, Journal of the American Chemical Society 135 (6) (2013) 2164-2171.

[9] G. H. Peters, M. Werge, M. N. Elf-Lind, J. J. Madsen, G. F. Velardez, P. Westh, Interaction of neurotransmitters with a phospholipid bilayer: a molecular dynamics study, Chemistry and physics of lipids 184 (2014) 7-17.

[10] S. Jo, T. Kim, V. G. Iyer, W. Im, CHARMM-GUI: A Web-Based Graphical User Interface for CHARMM., J. Comput. Chem. 29 (11) (2008) 1859-1865.

[11] S. Jo, J. B. Lim, J. B. Klauda, W. Im, CHARMM-GUI Membrane Builder for Mixed Bilayers and Its Application to Yeast Membranes., Biophys. J. 97 (1) (2009) 50-58.

[12] W. L. Jorgensen, J. Chandrasekhar, J. D. Madura, R. W. Impey, M. L. Klein, Comparison of Simple Potential Functions for Simulating Liquid Water, J. Chem. Phys. 79 (2) (1983) 926-935.

[13] J. C. Phillips, R. Braun, W. Wang, J. Gumbart, E. Tajkhorshid, E. Villa, C. Chipot, R. D. Skeel, L. Kalé, K. Schulten, Scalable Molecular Dynamics with NAMD., J. Comput. Chem. 26 (16) (2005) 1781-1802.

[14] J. L. Thewalt, M. Bloom, Phosphatidylcholine: cholesterol phase diagrams, Biophysical journal 63 (4) (1992) 1176-1181. 
[15] N. Kučerka, M.-P. Nieh, J. Katsaras, Fluid phase lipid areas and bilayer thicknesses of commonly used phosphatidylcholines as a function of temperature, BBA-Biomembranes 1808 (11) (2011) 2761-2771.

[16] H. J. Berendsen, J. v. Postma, W. F. van Gunsteren, A. DiNola, J. Haak, Molecular dynamics with coupling to an external bath, J. Chem. Phys. 81 (8) (1984) 3684-3690.

[17] J. B. Klauda, R. M. Venable, J. A. Freites, J. W. O'Connor, D. J. Tobias, C. Mondragon-Ramirez, I. Vorobyov, A. D. MacKerell, R. W. Pastor, Update of the CHARMM All-Atom Additive Force Field for Lipids: Validation on Six Lipid Types., J. Phys. Chem. B 114 (23) (2010) 7830-7843.

[18] J. B. Lim, B. Rogaski, J. B. Klauda, Update of the Cholesterol Force Field Parameters in CHARMM., J. Phys. Chem. B 116 (1) (2012) 203-210.

[19] U. Essmann, L. Perera, M. L. Berkowitz, T. Darden, H. Lee, L. G. Pedersen, A Smooth Particle Mesh Ewald Method, J. Chem. Phys. 103 (19) (1995) 8577-8593.

[20] I. Wood, M. F. Martini, M. Pickholz, Similarities and differences of serotonin and its precursors in their interactions with model membranes studied by molecular dynamics simulation, Journal of Molecular Structure 1045 (2013) 124-130.

[21] D. B. Kell, P. D. Dobson, S. G. Oliver, Pharmaceutical drug transport: the issues and the implications that it is essentially carrier-mediated only, Drug discovery today 16 (15-16) (2011) 704-714.

[22] L. Di, P. Artursson, A. Avdeef, G. F. Ecker, B. Faller, H. Fischer, J. B. Houston, M. Kansy, E. H. Kerns, S. D. Krämer, et al., Evidence-based approach to assess passive diffusion and carrier-mediated drug transport, Drug discovery today 17 (15-16) (2012) 905-912.

[23] O. Tsinman, K. Tsinman, N. Sun, A. Avdeef, Physicochemical selectivity of the bbb microenvironment governing passive diffusion?matching with a 
porcine brain lipid extract artificial membrane permeability model, Pharmaceutical research 28 (2) (2011) 337-363.

[24] P. R. Pandey, S. Roy, Headgroup mediated water insertion into the dppc bilayer: a molecular dynamics study, J. Phys. Chem. B 115 (12) (2011) $3155-3163$.

[25] H. I. Petrache, S. W. Dodd, M. F. Brown, Area per lipid and acyl length distributions in fluid phosphatidylcholines determined by $2 \mathrm{~h} \mathrm{nmr} \mathrm{spec-}$ troscopy, Biophys. J. 79 (6) (2000) 3172-3192.

[26] S. Chiu, E. Jakobsson, R. J. Mashl, H. L. Scott, Cholesterol-induced modifications in lipid bilayers: a simulation study, Biophys. J. 83 (4) (2002) $1842-1853$.

[27] C. Hofsäß, E. Lindahl, O. Edholm, Molecular dynamics simulations of phospholipid bilayers with cholesterol, Biophys. J. 84 (4) (2003) 2192-2206.

[28] O. Edholm, J. F. Nagle, Areas of molecules in membranes consisting of mixtures, Biophys. J. 89 (3) (2005) 1827-1832.

[29] Y. Wang, P. Gkeka, J. E. Fuchs, K. R. Liedl, Z. Cournia, Dppc-cholesterol phase diagram using coarse-grained molecular dynamics simulations, BBABiomembranes 1858 (11) (2016) 2846-2857.

[30] J. F. Nagle, S. Tristram-Nagle, Structure of lipid bilayers, BBA-Rev. Biomembranes 1469 (3) (2000) 159-195.

[31] D. Chandler, Introduction to Modern Statistical Mechanics, Oxford University Press, 1987.

[32] D. Trzesniak, A.-P. E. Kunz, W. F. van Gunsteren, A comparison of methods to compute the potential of mean force, ChemPhysChem 8 (1) (2007) $162-169$.

[33] P. L. Geissler, C. Dellago, D. Chandler, J. Hutter, M. Parrinello, Autoionization in liquid water, Science 291 (5511) (2001) 2121-2124. 
[34] J. Martí, F. S. Csajka, D. Chandler, Stochastic transition pathways in the aqueous sodium chloride dissociation process, Chem. Phys. Lett. 328 (1) (2000) 169-176.

[35] P. G. Bolhuis, D. Chandler, C. Dellago, P. L. Geissler, Transition path sampling: Throwing ropes over rough mountain passes, in the dark, Annu. Rev. Phys. Chem. 53 (1) (2002) 291-318.

[36] J. Martí, F. S. Csajka, Transition path sampling study of flip-flop transitions in model lipid bilayer membranes, Phys. Rev. E 69 (6) (2004) 061918.

[37] A. Barducci, G. Bussi, M. Parrinello, Well-tempered metadynamics: a smoothly converging and tunable free-energy method, Phys. Rev. Lett. $100(2)$ (2008) 020603.

[38] J. Yang, C. Calero, M. Bonomi, J. Martí, Specific ion binding at phospholipid membrane surfaces, J. Chem. Theor. Comput. 11 (9) (2015) 44954499.

395 [39] J. Martí, Analysis of the hydrogen bonding and vibrational spectra of supercritical model water by molecular dynamics simulations, J. Chem. Phys. 110 (14) (1999) 6876-6886.

[40] M. W. Feyereisen, D. Feller, D. A. Dixon, Hydrogen bond energy of the water dimer, J. Phys. Chem. 100 (8) (1996) 2993-2997.

400 [41] J. L. MacCallum, W. D. Bennett, D. P. Tieleman, Distribution of amino acids in a lipid bilayer from computer simulations, Biophysical Journal 94 (9) (2008) 3393-3404. 